\title{
CONHECIMENTO DE UM GRUPO DE PACIENTES SOBRE MEDICAMENTOS GENÉRICOS POR ELES UTILIZADOS
}

Antonio Carlos da Silva ${ }^{1}$, Luciana Regina Ferreira da Mata ${ }^{1}$, Paulo Celso Prado Telles Filho², José Fernando Petrilli Filho ${ }^{3}$

RESUMO: O objetivo deste estudo foi analisar o conhecimento de um grupo de pacientes de uma Santa Casa do interior do Estado de Minas Gerais acerca dos medicamentos genéricos por eles utilizados. Foram entrevistados, em domicílio, 25 pacientes que obtiveram alta hospitalar da Clínica Médica no mês de outubro de 2006. Os dados foram coletados no período de 18 de novembro de 2006 a 03 de janeiro de 2007, por meio de um questionário que versou sobre nome, dose, frequiência, horário, efeito esperado e efeito colateral, dentre outros. Constatou-se que 5 pacientes (20\%) desconheciam os nomes dos medicamentos que utilizam, $7(28 \%)$ a dose, $4(16 \%)$ a freqüência, 16 (24\%) o efeito esperado, 21 (84\%) o efeito colateral e 14 (56\%) não sabiam o que é medicamento genérico. Verificou-se um diminuto conhecimento acerca dos medicamentos genéricos, sendo necessárias amplas ações educativas por parte do enfermeiro.

PALAVRAS-CHAVE: Conhecimento; Enfermagem; Uso de medicamentos; Medicamentos genéricos.

\section{KNOWLEDGE OF A PATIENT GROUP ON GENERIC DRUGS THEY WERE TAKEN}

ABSTRACT: This study aimed to analyze the knowledge of a patient group at a philanthropic hospital in the interior of Minas Gerais State, Brazil, on generic drugs they were taking. Twenty-five patients discharged from the medical clinic in October 2006 were interviewed at home. Data collection was undertaken between November $18^{\text {th }}, 2006$ and January $03^{\text {rd }}, 2007$ through a questionnaire that asked the name, dose, frequency, time, expected effect and side-effect, among others. Five (20\%) patients did not know the name of the drug they were taking, 7 (28\%) did not know the dose, 4 (16\%) did not know the frequency, $16(24 \%)$ did not know the expected effect, $21(84 \%)$ did not know the side effect and $14(56 \%)$ did not know what generic drugs are. It was evidenced that patients have little knowledge on generic medication, which demands wide-ranging educational actions by nurses.

KEYWORDS: Knowledge; Nursing; Medication use; Generic drugs.

\section{CONOCIMIENTO DE UN GRUPO DE PACIENTES SOBRE MEDICAMENTOS GENÉRICOS UTILIZADOS POR ELLOS}

RESUMEN: La finalidad de este estudio fue analizar el conocimiento de un grupo de pacientes de un hospital filantrópico en el interior del Estado de Minas Gerais, Brasil, acerca de los medicamentos genéricos utilizados por ellos. Se entrevistaron en domicilio 25 pacientes, que recibieron alta hospitalaria de la Clínica Médica en octubre de 2006. Las entrevistas fueron realizadas en el período entre 18 de noviembre de 2006 y 03 de enero de 2007, por medio de un cuestionario que abarcó nombre, dosis, frecuencia, horario, efecto esperado y efecto colateral, entre otros. Se destacó que 5 (20\%) desconocen el nombre del medicamento que utilizan; 7 (28\%) desconocen la dosis; 4 (16\%), la frecuencia; 16 (24\%), el efecto esperado; 21 (84\%), el efecto colateral; y 14 (56\%) no saben qué es medicamento genérico. Se verificó un reducido conocimiento acerca de los medicamentos genéricos, siendo necesarias acciones educativas amplias por parte del enfermero.

PALBRAS CLAVE: Conocimiento; Enfermería; Uso de medicamentos; Medicamentos genéricos.

\footnotetext{
${ }^{1}$ Acadêmico. Curso de Graduação em Enfermagem da Universidade Federal dos Vales do Jequitinhonha e Mucuri - UFVJM.

${ }^{2}$ Professor Adjunto 1. Departamento de Enfermagem. UFVJM. Doutor pela Escola de Enfermagem de Ribeirão Preto. Universidade de São Paulo - EERP-USP

${ }^{3}$ Professor Assistente 2. Departamento de Enfermagem. Universidade de São Carlos - UFSCar. Doutorando pela EERP-USP.
}

Autor correspondente:

Paulo Celso Prado Telles Filho

Rua Tiradentes, 150 - 39100-000 - Diamantina-MG

Recebido: 08/03/07

E-mail: ppradotelles@yahoo.com.br

Aprovado: 22/10/07 


\section{INTRODUÇÃO}

Após a segunda Guerra Mundial, a indústria farmacêutica conheceu um período de grande desenvolvimento. Integrada aos novos mecanismos de produção e mercado, tornou-se um dos segmentos mais lucrativos da produção industrial contemporânea ${ }^{(1)}$. Esse crescimento foi de primordial importância para o desenvolvimento quantiqualitativo de medicamentos, tornando-os mais eficazes. No entanto, foi necessário um investimento mais aguçado na produção e na promoção das vendas, elevando assim, de maneira considerável, o custo financeiro.

Existe um paradoxo no consumo mundial de medicamentos: de um lado tem-se a criação de medicamentos de alta tecnologia, mais potentes e de ação cada vez mais específica e, de outro, uma parcela significativa da população mundial sem acesso a medicamentos essenciais ${ }^{(2)}$.

No Brasil uma das causas que dificultam a utilização de medicamentos está relacionada ao seu custo, pois grande parte da população não possui condição financeira para a sua aquisição e não tem total acesso a medicamentos distribuídos pelo governo. Deve-se, ainda, levar em consideração que, devido a dificuldades de acesso a serviços de saúde, muitos indivíduos necessitam da utilização de vários medicamentos.

$\mathrm{Na}$ implementação de políticas nacionais de medicamentos recomendadas pela Organização Mundial da Saúde (OMS), vários países incentivam o uso de medicamentos genéricos como estratégia importante para fomentar preços acessíveis e, conseqüentemente, o próprio acesso aos medicamentos ${ }^{(3)}$.

A política de medicamentos genéricos objetiva também uma maior racionalidade em sua utilização, estimula a concorrência à medida que os consumidores possuem disponíveis produtos intercambiáveis de diferentes preços, respeitando-se a decisão de não intercambialidade do profissional prescritor ${ }^{(4)}$.

Os medicamentos genéricos foram implantados com o intuito de aumentar o acesso da população aos medicamentos, uma vez que são mais acessíveis financeiramente; isso se deve ao fato de sua fabricação não necessitar de altos investimentos em pesquisas, uma vez que são utilizados os medicamentos de referência como parâmetro; além disso, não há gastos com propagandas, já que não existe marca a ser divulgada. Verifica-se, portanto, que os medicamentos genéricos possuem o mesmo princípio ativo, dose, fórmula farmacêutica, via de administração e mesma indicação terapêutica do medicamento de referência, porém com menor custo.

Estudos desenvolvidos abrangem principalmente a biodisponibilidade dos fármacos, a bioequivalência com o medicamento de referência e as dimensões de impacto que os medicamentos genéricos têm causado no mercado farmacêutico. No entanto, sente-se uma lacuna em relação às implicações sociais que o medicamento genérico tem causado na população, principalmente no que diz respeito à confiabilidade e aceitabilidade desses produtos pela sociedade ${ }^{(4)}$. Portanto, é necessário que exista uma expansibilidade na tarefa pedagógica do enfermeiro, pois cabe a ele informar e educar sobre os aspectos aqui descritos.

$\mathrm{O}$ processo da administração de medicamentos exige conhecimento atualizado ${ }^{(5)}$. As exigências em relação à administração de medicamentos são crescentes, havendo consenso entre pesquisadores e profissionais envolvidos com a Enfermagem de que o conhecimento ocupa papel de destaque entre essas exigências ${ }^{(6)}$. Portanto, torna-se necessário destacar a importância do enfermeiro, bem como da equipe de enfermagem na educação do paciente em relação a esse tema.

Dessa forma, o presente estudo é relevante à medida que pretende analisar o conhecimento de pacientes acerca dos medicamentos genéricos, com vistas a uma segurança mais ampla na manutenção da terapêutica medicamentosa, uma vez que o conhecimento é um aspecto fundamental no processo relacionado a tal terapêutica. Assim, neste estudo tem-se como objetivo analisar o conhecimento de um grupo de pacientes de uma Santa Casa do interior do Estado de Minas Gerais acerca dos medicamentos genéricos por eles utilizados.

\section{DESCRIÇÃO DO MÉTODO}

Trata-se de um estudo quantitativo descritivo. O método quantitativo caracteriza-se pelo emprego da quantificação, tanto nas modalidades de coleta de informações, quanto no tratamento delas por meio de técnicas estatísticas. Os estudos de natureza descritiva propõem-se a descobrir as características de um fenômeno como tal. Nesse sentido, são considerados como objeto de estudo uma situação específica, um grupo ou um indivíduo ${ }^{(7)}$.

O estudo foi realizado na Santa Casa de um município do interior do Estado de Minas Gerais, uma 
instituição de caráter filantrópico, sem fins lucrativos, fundada em 23 de maio de 1790. Conta com 73 leitos e a equipe de enfermagem é formada por 4 enfermeiros, 11 técnicos e 50 auxiliares de enfermagem. A clínica médica, local do presente estudo, possui 29 leitos, conta com um quadro de pessoal de enfermagem formado por 2 enfermeiros, 11 técnicos e 15 auxiliares de enfermagem e atende às seguintes especialidades: hemodiálise, oftalmologia, cardiologia, nefrologia, neurologia, ortopedia/traumatologia, geriatria, otorrinolaringologia, dermatologia e clínica geral.

Foram entrevistados indivíduos adultos, de ambos os sexos, conscientes, orientados no tempo e espaço, residentes na zona urbana do município em que se localiza a instituição hospitalar em estudo e que obtiveram alta hospitalar no mês de outubro de 2006 da clínica descrita. As entrevistas foram realizadas em domicílio e foram excluídos os que se recusaram a participar, bem como aqueles que não puderam prestar informações verbais. Sendo assim, este estudo foi realizado com 25 pacientes.

Mediante um levantamento realizado na seção de arquivo da instituição investigada, por meio de registros hospitalares, foram identificados pacientes que obtiveram alta hospitalar no mês de outubro de 2006 e que possuíam prescrições de medicamentos genéricos. Pelos registros dos prontuários identificaram-se os endereços domiciliares, realizando-se a coleta de dados, por meio de visitas domiciliares realizadas no período de 18 de novembro de 2006 a 03 de janeiro de 2007. É importante destacar que anteriormente à visita era realizado um telefonema explicados os objetivos da pesquisa, solicitado o aceite em participar, bem como agendado horário mais conveniente tanto para os pesquisadores como para o paciente.

Utilizou-se um questionário que incluía variáveis que faziam referência à identificação do paciente (idade, sexo, estado conjugal, escolaridade, diagnóstico médico), ao medicamento (nome, dose, freqüência, horário, efeito esperado, efeito colateral), ao conhecimento do paciente sobre medicamentos genéricos, à diferença e identificação entre medicamentos de referência, similar e genérico, ao tipo de atendimento na internação (público, privado ou plano de saúde), ao recebimento de instruções, à confiança e ao conhecimento sobre a legislação referente aos medicamentos genéricos.

Os dados obtidos através desse questionário, foram tabulados e armazenados no Programa Microsoft Excel. Como tratamento estatístico, optouse pela utilização da estatística descritiva e posterior discussão, a partir de literatura atualizada. Para descrever o perfil da amostra, segundo as variáveis acima citadas, foram elaborados quadros contendo frequiências absolutas e relativas.

O estudo foi submetido à análise da Direção Clínica da Instituição Hospitalar em que o mesmo foi realizado. Foi iniciado após a aprovação pelo Comitê de Ética em Pesquisa da Universidade Federal dos Vales do Jequitinhonha e Mucuri UFVJM, sob o parecer n. 067/06 de 26/09/2006. Cada participante assinou o Termo de Consentimento Livre e Esclarecido para Participação em Pesquisa, quando foi esclarecido o objetivo do estudo e que poderia se recusar a participar ou se excluir quando e se julgasse necessário. A cada participante foi também garantido o direito do anonimato, ficando claro que não haveria desconforto, danos e/ou risco e também que poderia deixar de responder qualquer pergunta que the causasse constrangimento de qualquer natureza.

\section{RESULTADOS E DISCUSSÃO}

Anteriormente aos resultados, apresenta-se a identificação dos participantes deste estudo. Em relação à idade, tem-se que a idade mínima foi 18 anos e a máxima 91 anos, com média de 60,44 e mediana 63. Desses participantes, 7 (28\%) eram do sexo feminino e 18 (72\%) do sexo masculino. Quanto ao estado civil, 3 (12\%) eram solteiros, 16 (64\%) casados, 5 (20\%) viúvos e 1 (4\%) separado. Em relação ao grau de escolaridade, 10 (40\%) eram analfabetos, 3 (12\%) semi-analfabetos, 8 (32\%) possuíam ensino fundamental incompleto, $3(12 \%)$ ensino fundamental completo e $1(4 \%)$ ensino médio completo. Quanto ao diagnóstico médico, 3 (12\%) o desconheciam, 12 (48\%) referiram hipertensão arterial, 3 (12\%) pneumonia, 2 (8\%) infecção renal, 2 (8\%) bronquite, 1 (4\%) constipação intestinal, 1 (4\%) reumatismo e 1 (4\%) câncer de próstata.

Por meio da coleta dos dados, verificou-se que existe importante déficit de conhecimento acerca dos medicamentos na amostra em estudo, como pode ser observado no Quadro 1.

Nesse Quadro importa destacar que dentre os 25 pacientes questionados, 5 (20\%) relataram desconhecer o nome do medicamento que utilizavam, 7 $(28 \%)$ desconheciam a dose e $4(16 \%)$ desconheciam a 
Quadro 1 - Distribuição dos pacientes em relação ao conhecimento dos medicamentos genéricos por eles utilizados, 2007

\begin{tabular}{|lcccc|}
\hline Conhecimento Conhece Desconhece & $\begin{array}{c}\text { Conhece } \\
\text { parcialmente }\end{array}$ & Total \\
\hline Nome & 19 & 5 & 1 & 25 \\
& $(76 \%)$ & $(20 \%)$ & $(4 \%)$ & $(100 \%)$ \\
Dose & 15 & 7 & 3 & 25 \\
& $(60 \%)$ & $(28 \%)$ & $(12 \%)$ & $(100 \%)$ \\
Freqüência & 20 & 4 & 1 & 25 \\
& $(80 \%)$ & $(16 \%)$ & $(4 \%)$ & $(100 \%)$ \\
Horário & 21 & 4 & - & 25 \\
& $(84 \%)$ & $(16 \%)$ & & $(100 \%)$ \\
Efeito esperado & 14 & 6 & 5 & 25 \\
& $(56 \%)$ & $(24 \%)$ & $(20 \%)$ & $(100 \%)$ \\
Efeito colateral & 1 & 21 & 3 & 25 \\
& $(4 \%)$ & $(84 \%)$ & $(12 \%)$ & $(100 \%)$ \\
\hline
\end{tabular}

frequiência no uso desses medicamentos. Quanto ao horário de administração, 4 (16\%) referiram desconhecê-lo. Diante da ação do medicamento, 6 (24\%) narraram desconhecer o efeito esperado e 21 (84\%) disseram desconhecer o efeito colateral. É importante citar que se considerou a opção "conhece parcialmente" para aqueles que apresentaram conhecimento a respeito de determinado medicamento e desconhecimento para outro.

O desconhecimento do nome e da dose utilizada pelos pacientes pode contribuir para agravar seu estado clínico, uma vez que pode minimizar a efetividade do tratamento, pois uma dose e um medicamento diferente daquele prescrito pode acentuar os efeitos colaterais, bem como não produzir o efeito esperado.

A resposta do paciente a um determinado medicamento reflete a quantidade da dose administrada. De modo peculiar, à medida que a dose aumenta, a resposta farmacológica aumenta de maneira gradual e contínua até que os receptores se tornem plenamente ocupados pela substância. Nesse ponto, aumentar a dose não estimularia a resposta, mas aumentaria o risco de reações adversas ${ }^{(8)}$.

Quanto ao horário, é importante que durante a prescrição e instrução sobre o medicamento seja respeitado o modo de vida do paciente, levando em consideração o melhor horário para ser administrada a medicação.

Reconhecidos como instrumentos indispensáveis às ações de saúde, os medicamentos ocupam papel central na terapêutica da atualidade. Fatores relacionados ao modo de utilização refletem-se no efeito colateral e terapêutico e, por isso, nem sempre exercem plenamente o seu papel. Promover o uso racional de medicamentos é um desafio que depende de vários fatores, dentre eles, educação e informação adequada às equipes de saúde e consumidores ${ }^{(9)}$.

Outros fatores relevantes são as informações contidas nos textos das bulas dos medicamentos, pois na maioria das vezes este é o único meio de informação acessível ao paciente, já que nem sempre é possível o acesso a um profissional capacitado para o esclarecimento de dúvidas. Entretanto grande parte das bulas são consideradas insatisfatórias e apenas cumprem a obrigatoriedade legal de serem inseridas na embalagem.

A bula poderia servir como fonte confiável e adequada de informação, suprindo em parte a carência informacional; entretanto, não é capaz de fazê-lo satisfatoriamente por não cumprir inteiramente seu papel. Para que as informações contidas nas bulas sejam úteis e cumpram sua finalidade, devem ser apresentadas de maneira objetiva e atualizada, em consonância com a legislação vigente e o conhecimento técnicocientífico atual, não devendo apresentar informações distorcidas aos que prescrevem e utilizam o medicamento ${ }^{(10)}$.

Diante desses dados observa-se uma necessidade de incrementar atitudes que facilitem e maximizem o conhecimento dos pacientes em relação aos medicamentos por eles utilizados, principalmente em relação aos efeitos colaterais, à dose e aos efeitos esperados, pontos de fundamental importância para a adesão e para o bom desempenho do processo terapêutico.

No que concerne ao conhecimento sobre o que são os medicamentos genéricos, 14 pacientes $(56 \%)$ disseram não conhecer, $2(8 \%)$ relataram que é o mesmo medicamento comparado ao medicamento de referência, faz o mesmo efeito e é mais barato, enquanto 7 (28\%) responderam ser o medicamento mais barato e $2(8 \%)$ mencionaram ser mais barato e mais fraco, conforme os relatos:

\section{É o remédio que é mais barato.}

É o remédio que é mais barato, mas é mais fraco que os outros.

O baixo nível de conhecimento, bem como conceitos errôneos sobre os medicamentos genéricos, devem-se principalmente à deficiência de informações sobre o uso dos genéricos e ao baixo nível de 
escolaridade da amostra em estudo; ressalta-se, também, a deficiência na política de divulgação, principalmente no início da implantação dos medicamentos genéricos no Brasil.

Um dos desafios da política de genéricos está em transmitir ao consumidor as informações necessárias para que este se torne, de fato, um usuário desses medicamentos. O paciente possui poucas condições de escolher por si só qual medicamento vai adquirir, tornandose dependente de opinião profissional. Dessa maneira, o principal canal de estímulo aos medicamentos genéricos está na contínua educação sobre a disponibilidade, os princípios ativos, o diferencial de preços e a eficácia idêntica desse grupo de medicamentos ${ }^{(11)}$.

Em relação às categorias de medicamentos, 25 pacientes (100\%) desconheciam a diferença entre medicamentos de referência, similares e genéricos. Esse desconhecimento destacou-se nesse estudo, uma vez que se trata de conceito básico e essencial para a utilização segura dos medicamentos. Como exemplo de resposta a esse item, transcreve-se o seguinte trecho:

\section{O genérico é importado e o de referência e similar não.}

Estes dados concordam com pesquisa realizada no ano de 2004, pela Associação Brasileira das Indústrias de Medicamentos Genéricos (Pró-Genéricos), com amostra de 900 consumidores de quatro capitais brasileiras, a qual identificou que $30 \%$ dos consumidores desconheciam o que era medicamento similar e $78 \%$, medicamento de referência e apenas 19\% mencionaram o genérico como primeira opção para compra ${ }^{(12)}$.

Quanto à identificação entre medicamentos de referência, similares e genéricos, 17 pacientes (68\%) não souberam identificar e $8(32 \%)$ relataram saber identificar o genérico devido à tarja amarela na embalagem.

O baixo conhecimento observado sobre medicamentos genéricos indica a necessidade de revisão global do programa pelas medidas educativas até então praticadas e maior envolvimento das instituições e de seus agentes, visando boas práticas de assistência terapêutica ${ }^{(13)}$.

Em relação ao tipo de atendimento prestado durante a internação hospitalar, tem-se que 20 pacientes $(80 \%)$ tiveram atendimento público, $2(8 \%)$ particular e $3(12 \%)$ com plano de saúde. Destaca-se a gravidade dessa ocorrência, pois se infere que os pacientes atendidos pelo sistema público são os de menor poder aquisitivo e, portanto, aqueles que necessitariam de medicamentos menos onerosos.
A esse respeito, os dispêndios efetuados pelas famílias com saúde são o quarto grupo mais volumoso das despesas de consumo familiar, seguido apenas das despesas efetuadas com habitação, alimentação e transporte. Entre os menos favorecidos economicamente, o gasto em saúde é majoritariamente realizado com a compra de medicamentos ${ }^{(14)}$.

O Quadro 2, apresenta dados referentes à instrução e confiança em tais medicamentos e ao conhecimento sobre a lei dos medicamentos genéricos.

Quadro 2 - Distribuição dos pacientes quanto à instrução, confiança e ao conhecimento sobre os medicamentos genéricos, 2007

\begin{tabular}{|lccc|}
\hline Categorias & Sim & Não & Total \\
\hline Recebeu Instrução sobre & 1 & 24 & 25 \\
Medicamentos Genéricos & $(4 \%)$ & $(96 \%)$ & $(100 \%)$ \\
Confia nos Medicamentos & 9 & 16 & 25 \\
Genéricos & $(36 \%)$ & $(64 \%)$ & $(100 \%)$ \\
Tem conhecimento sobre & 3 & 22 & 25 \\
a Lei dos Genéricos & $(12 \%)$ & $(88 \%)$ & $(100 \%)$ \\
\hline
\end{tabular}

Destaca-se que, quanto à instrução sobre os medicamentos genéricos, 24 pacientes $(96 \%)$ relataram não recebê-la, 16 (64\%) disseram não confiar nessa categoria de medicamentos e $22(88 \%)$ alegaram ainda não ter conhecimento sobre a lei dos genéricos.

Em relação à instrução, é importante ressaltar que, além de permitir maior acesso da população aos medicamentos e melhorar o processo de adesão à terapêutica, ela propicia também a conscientização contra a automedicação, uma importante ocorrência observada na prática assistencial.

Informar sobre a segurança e a qualidade dos medicamentos genéricos tem o efeito instrucional de apontar para a responsabilidade necessária ao lidar com medicamentos, que são drogas a serem utilizadas apenas com a recomendação de profissionais especializados. A existência dos medicamentos genéricos auxilia a combater a visão de que os medicamentos são um produto de consumo qualquer, e que as pessoas podem entrar numa farmácia e comprar o que quiserem, quando acharem conveniente. A lei dos medicamentos genéricos abre todas as possibilidades para que os princípios democráticos dos direitos do consumidor se tornem realidade na relação dos brasileiros com os medicamentos ${ }^{(15)}$.

No entanto, para que o obstáculo do desconhecimento seja superado, é necessário que haja uma interação eficiente entre os enfermeiros, equipe 
de enfermagem e pacientes, socializando informações corretas e baseadas em fontes científicas seguras, não desvalorizando, contudo, os conceitos socioeconômicos e culturais do paciente.

Quanto ao desconhecimento encontrado sobre a lei dos medicamentos genéricos, verifica-se que grande parte dos pacientes não possui informação sobre seus direitos legais em relação ao consumo de medicamentos, ao mesmo tempo em que revela a pouca eficácia no processo de divulgação dessa lei. Esses déficits podem estar relacionados à ocorrência de erros de medicação em domicílio. A esse respeito tem-se que tal ocorrência está relacionada ao trabalho do enfermeiro, uma vez que é da competência desse profissional instruir o paciente para o uso correto dos medicamentos, após a alta hospitalar ${ }^{(16)}$.

$\mathrm{O}$ resultado relacionado à confiança nos medicamentos está intimamente ligado à desinformação dos pacientes em relação a essa categoria medicamentosa. Os pacientes necessitam ser fortemente estimulados a comentar e esclarecer dúvidas, possibilitando a aquisição dos conhecimentos necessários para o uso dos medicamentos de forma segura e racional ${ }^{(17)}$. Portanto, é de extrema importância que pacientes tenham informações confiáveis acerca dos medicamentos por eles utilizados, pois assim haverá maior adesão deles ao plano terapêutico prescrito, culminando com um tratamento adequado. Quanto mais efetivo for o processo de produção de conhecimento no que concerne aos medicamentos e quanto melhor a interação entre equipe de saúde e paciente, mais próximo se está de alcançar um melhor resultado terapêutico ${ }^{(18)}$.

\section{CONSIDERAÇÕES FINAIS}

A partir dos dados analisados neste estudo detectou-se um importante déficit de conhecimento de pacientes acerca dos medicamentos genéricos por eles utilizados, principalmente no que se refere aos efeitos colaterais, efeitos esperados, bem como ao próprio nome do medicamento. Essa constatação é de grande gravidade, uma vez que os pacientes necessitam possuir conhecimento de todos os aspectos relacionados à terapêutica medicamentosa, como nomes, indicação, efeitos colaterais, horários, tempo de utilização e dosagem. Portanto, é pertinente que haja o esclarecimento desses aspectos ao paciente, pois muitos indivíduos aderem parcialmente ou mesmo não aderem à terapêutica prescrita, devido ao escasso conhecimento. Assim, vale ressaltar a importância do papel do enfermeiro, bem como da equipe de enfermagem no processo da medicação. É imprescindível que esses profissionais disponibilizem seus conhecimentos, durante a orientação aos pacientes, de forma adequada e segura.

Espera-se que este estudo contribua para alertar sobre o déficit de conhecimento verificado neste grupo de pacientes, destacar a importância da qualidade da orientação que a equipe necessita oferecer aos pacientes sob sua responsabilidade e enfatizar que a aliança entre Enfermagem e Educação pode e deve contribuir, auxiliando a construir um processo de saúde com nível de excelência para a sociedade.

Ressalta-se, também, a extrema importância de aprofundar a discussão sobre o papel do enfermeiro na efetiva orientação à sociedade quanto aos medicamentos utilizados, pois através de sua atuação educativa, haverá um mais profundo conhecimento sobre os medicamentos utilizados e conseqüentemente uma otimização da terapêutica.

É necessário que enfermeiros, pacientes e sociedade se sintam convidados a superar modelos em que se admitia um conhecimento superficial, para avançar em direção a práticas mais efetivas no contexto da administração de medicamentos.

\section{REFERÊNCIAS}

1 Nascimento MC. Medicamentos, comunicação e cultura. Ciênc saúde coletiva. 2005;10(sup):179-193.

2 Dias CRC. Medicamentos genéricos no Brasil de 1999 a 2002: análise da legislação, aspectos conjunturais e políticos [dissertação]. São Paulo (SP): Faculdade de Saúde Pública da Universidade de São Paulo; 2003.

3 Carvalho MCRD, Accioly Júnior H, Raffin FN, Campos MN, Cruz MMC, Alves MKS. Representações sociais do medicamento genérico por farmacêuticos: determinação do sistema central e periférico. Cad Saude Publ. 2006;21(1):226-34.

4 Carvalho MCRD, Accioly Júnior H, Raffin FNR. Representações sociais do medicamento genérico por consumidores residentes em Natal, Rio Grande do Norte, Brasil. Cad Saúde Publ. 2006;22(3):653-61.

5 Telles Filho PCP, Cassiani SHB. Administração de medicamentos: necessidades educacionais de enfermeiros e proposição de um curso de atualização. 
Cogitare Enferm. 2001Jul/Dez; 6(2):74.

6 Telles Filho PCP, Cassiani SHB. Utilização da Plataforma Teleduc no ensino da Administração de Medicamentos em um Curso de Graduação em Enfermagem: a busca pela excelência. Cogitare Enferm. 2006 Mai/Ago;11(2):178.

7 Richardson RJ. Pesquisa social: métodos e técnicas. $3^{\mathrm{a}}$ ed. São Paulo: Atlas; 2007.

8 Cabral IE. Administração de medicamentos. Rio de Janeiro: Reichmann e Affonso Editores; 2002.

9 Lage EA, Freitas MIF, Acurcio FA. Informação sobre medicamentos na imprensa: uma contribuição para o uso racional? Ciênc Saúde Coletiva. 2005;10(sup):133-9.

10 Gonçalves SA, Melo G, Tokarski MHL, Barbosa-Branco A. Bulas de medicamentos como instrumento de informação técnico-científica. Rev Saúde Publ. 2002;36(1):33-9.

11 Monteiro WM, Melo GC, Massunari GK, Hubner DV, Tasca RS. Avaliação da disponibilidade de medicamentos genéricos em farmácias e drogarias de Maringá (PR) e comparação de seus preços com os de referência e similares. Rev Bras Ciências Farmac. 2005;41(3):333-43.

12 Rumel D, Nishioka SA, Santos AAM. Intercambialidade de medicamentos: abordagem clínica e o ponto de vista do consumidor. Rev Saúde Publ. 2006;40(5):921-7.

13 Faria MAS, Tavares-Neto J. Conhecimento popular sobre medicamento genérico em um distrito docenteassistencial do município de Rio Branco, Estado do Acre, Brasil. Epidemiol Serv Saúde. 2006;15(3):37-45.

14 Silveira FG, Osório RG, Piola SF. Os gastos da família com saúde. Ciênc Saúde Coletiva. 2002;7(4):719-31.

15 Ministério da Saúde (BR). Agência Nacional de Vigilância Sanitária-ANVISA. Medicamentos genéricos: Brasília; 2002.

16 Miasso AI, Cassiani SHB. Administração de medicamentos: orientação final de enfermagem para a alta hospitalar. Rev Esc Enferm USP. 2005;39(2):13644.

17 Tierlin VL, Paulino MA, Fernandes LC, Schenkel EP, Mengue SS. Nível de conhecimento sobre a composição de analgésicos com ácido acetilsalicílico.
Rev Saúde Publ. 2000;38(2):223-7.

18 Pepe VLE, Castro CGSO. A interação entre prescritores, dispensadores e pacientes: informação compartilhada como possível benefício terapêutico. Cad Saúde Publ. 2000;16(3):815- 22. 